\title{
Análise de Vídeos como Recurso Educacional em Plataforma Não Formal de Aprendizagem
}

\author{
João Pedro Ferreira ${ }^{1}$, Pedro de Torres Maschio ${ }^{3}$, Thalia Santos de Santana ${ }^{2}$, \\ Newarney Torrezão da $\operatorname{Costa}^{1}$, Cleon Pereira Junior ${ }^{1}$ \\ ${ }^{1}$ Instituto Federal Goiano (IF Goiano) - Campus Iporá - Iporá-GO - Brasil \\ ${ }^{2}$ Instituto Federal Goiano (IF Goiano) - Campus Ceres - Ceres-GO - Brasil \\ ${ }^{3}$ Universidade de Brasília - Campus Darcy Ribeiro - Brasília-DF - Brasil \\ \{cleon.junior, newarney.costa\}@ifgoiano.edu.br, \\ \{pedrobarros2010, thaliassantana15, pedromaschio.ptm\}egmail.com
}

\begin{abstract}
The growing use of platforms for educational purposes, but which are not designed for this purpose, poses challenges in the analysis of the most appropriate form of content delivery. This paper highlights existing correlations between the IEEE-LOM Learning Object (OA) metadata and educational video metadata from the computer programming area, available on the Youtube platform. The outcomes showed a positive and moderate correlation between semantic density and difficulty, and a correlation between the frequency of nouns and semantic density. It was also evidenced a neutral correlation between likes, unlikes, views and comments with LOs analysed.
\end{abstract}

Resumo. O crescente uso de plataformas com fins educacionais, mas que não são concebidas para tal, impõe desafios na análise da forma mais adequada de entrega do conteúdo. Este trabalho evidencia correlações existentes entre os metadados de Objetos de Aprendizagem (OA) do padrão IEEE-LOM e metadados de vídeos educacionais da área de programação de computadores, disponibilizados na plataforma Youtube. Os resultados apresentaram uma correlação positiva e moderada entre densidade semântica e dificuldade, bem como uma correlação entre a frequência de substantivos e a densidade semântica. Também foi possível verificar que há uma correlação neutra de curtidas, descurtidas, visualizações e comentários com os metadados de OAs analisados.

\section{Introdução}

O processo de aprendizagem formal por muito tempo ocorreu por meio da sala de aula, com currículos e fontes de busca delimitados para obtenção de um conhecimento específico. Com o advento da Web e das mídias sociais, os meios para a aquisição de conhecimentos têm ampliado. É crescente o interesse de tutores e estudantes por buscas de materiais educacionais em fontes que não são voltadas para a aprendizagem [Westerhout et al. 2010], sendo a representação desses materiais discutida como aprendizagem não formal. Assim, esse conceito se refere ao aprendizado adaptado por meio de atividades planejadas, mas que não são explicitamente desenvolvidas para o contexto educacional [Cedefop 2014]. Dessa forma, mostra-se emergente a realização de investigações 
com relação aos materiais em fontes não formais, a fim de identificar soluções que possam beneficiar o processo de aprendizagem.

Na Web é possível encontrar podcasts, vídeos, imagens, textos, dentre outros formatos digitais que estão armazenados em repositórios que não foram desenvolvidos com propósitos educacionais. O Youtube ${ }^{1}$ e o Spotify ${ }^{2}$ são exemplos de plataformas que armazenam mídias digitais no formato de vídeo e áudio e que, apesar de não terem como exclusividade conteúdos educacionais, é possível vê-los sendo utilizados para essa finalidade [Bhabad et al. 2017, Andriani et al. 2018]. Por outro lado, os repositórios de Objetos de Aprendizagem (OA) e de Recursos Educacionais Abertos (REA) possuem mídias que foram desenvolvidas com o intuito específico de aprendizagem.

Uma das vantagens de utilizar um repositório de OA é que, além do conteúdo armazenado ter o foco na educação, estes costumam seguir um padrão para preenchimento dos metadados. Se um repositório armazenar os OA seguindo o padrão do IEEE-LOM, por exemplo, além de dados básicos como nome, formato, idioma, também existem informações educacionais como a dificuldade de aprender o conteúdo por intermédio daquele OA, o nível de interatividade, a densidade semântica, dentre outros [McClelland 2003]. Realizar uma busca partindo do pressuposto que os metadados do OA estão preenchidos permite personalizar o retorno de recursos educacionais, levando em consideração as necessidades do estudante.

Em uma plataforma cujo foco não é educacional, as mídias armazenadas e disponíveis não seguem padrões de OA, mas possuem metadados que podem ser relevantes para seleção de conteúdos. Geralmente essas plataformas disponibilizam a quantidade de visualizações, os comentários, as reações dos usuários (em forma de curtidas), dentre outros. No Youtube, por exemplo, além de dados estatísticos baseados nas experiências do usuário, é possível verificar a categoria do vídeo, palavras-chave e sua descrição.

Apesar de não serem ambientes educacionais, muitas plataformas da Web podem trazer conteúdos relevantes para a aprendizagem e até abranger uma quantidade maior de conceitos para os discentes quando comparadas com repositórios de OA [De Medio et al. 2019]. Nesse aspecto, surge a necessidade de investigar mídias para o processo de aprendizagem em um ambiente não educacional. Uma das possibilidades de investigação é analisar os dados fornecidos por estas plataformas e relacionar com informações que são relevantes ao processo de aprendizagem.

Com o objetivo de colaborar com pesquisas em Informática na Educação (IE) e aprendizagem baseada em vídeo, este trabalho apresenta uma análise de vídeos da plataforma Youtube, buscando correlações dos metadados fornecidos pela plataforma com metadados da categoria educacional do padrão IEEE-LOM. Para tal, uma base de dados foi construída considerando o domínio de programação de computadores.

\section{Fundamentação Teórica e Trabalhos Correlatos}

Entende-se por metadados como um conjunto de dados que fornecem informações resumidas acerca de alguma entidade, ou seja, dados sobre dados [Pal et al. 2019]. Os Metadados de OA, cujo termo em inglês é Learning Object Metadata, são formas de

\footnotetext{
${ }^{1}$ www.youtube.com

${ }^{2}$ www.spotify.com
} 
IX Congresso Brasileiro de Informática na Educação (CBIE 2020)

Anais do XXXI Simpósio Brasileiro de Informática na Educação (SBIE 2020)

representação dos dados de um OA. A IEEE, ainda em 2002, criou o padrão IEEE-LOM, adotado mundialmente para a representação de metadados de OA [McClelland 2003].

O IEEE-LOM estrutura os dados de um OA em nove categorias. Na categoria educacional é possível armazenar dados como tipo de recurso de aprendizagem, tipo de interatividade, nível de interatividade, densidade semântica, dificuldade, idioma, dentre outros [McClelland 2003]. Ao registrar um OA em um repositório, é necessário informar tais metadados. Contudo, o trabalho manual para preenchimento de metadados pode demandar bastante tempo [García-Floriano et al. 2017].

A quantidade de OA disponíveis em repositórios é limitada, assim, a recuperação de conteúdo Web é uma realidade que pode oferecer maior suporte em relação aos recursos de aprendizagem ofertados [De Medio et al. 2019]. A recuperação de recursos não estruturados e, posterior adequação em OA anotados, aumentam as chances de determinados conteúdos se tornarem mais relevantes durante o processo de recomendação em um ambiente de aprendizagem. Portanto, a análise de mídias não estruturadas deve ser levada em consideração para um possível preenchimento automático de metadados de OA.

A aprendizagem baseada em vídeo pode proporcionar um estudo mais individualizado e flexível. Além disso, tal recurso educacional pode ser enriquecido com artefatos que favorecem a memorização [Pal et al. 2019]. Neste sentido, pesquisas recentes centram esforços na recomendação de vídeos educacionais, em ambientes virtuais de aprendizagem, a partir de repositórios definidos [Barrére et al. 2018]. Por outro lado, observa-se que, em relação a este tipo de material, discentes e especialistas podem apresentar pontos de vista distintos [De Lima Medeiros and Pansanato 2015].

O trabalho de [Pal et al. 2019] apresenta uma abordagem semiautomática para classificação de vídeos como OAs seguindo o padrão IEEE-LOM. A partir de uma ontologia desenvolvida para o domínio de programação em linguagem Java, os autores buscaram classificar de forma automática os vídeos, informando parcialmente os metadados presentes no padrão IEEE-LOM. Deste modo, foi explorada a narração dos vídeos e termos presentes na ontologia para obter parte dos metadados de OA. Em comparação com uma base preenchida manualmente, a abordagem apresentou resultados satisfatórios para o domínio específico.

Em [Bhabad et al. 2017] é apresentado um sistema de recomendação de imagens, vídeos e páginas Web, a partir de metadados extraídos de vídeo. Nesse trabalho são exploradas técnicas de extração de metadados com base em imagens e textos, obtidos, respectivamente, a partir da segmentação do vídeo e análise da narração. A validação desse processo ocorreu com o julgamento por pares da relevância do conteúdo recomendado. No trabalho também utilizaram similaridade de cosseno e correlação de Pearson.

Diante do cenário exposto, nota-se que há investigações tanto no aspecto de busca de materiais em ambientes não educacionais quanto no âmbito de extração automática de metadados em vídeos, pois essas mídias têm sido bastante exploradas no processo de aprendizagem. Apesar dos trabalhos existentes apresentarem bastante avanço no estado da arte, ainda há uma carência na análise de correlações dos metadados de mídias em plataformas não educacionais com recursos de aprendizagem. Visando investigar possíveis correlações, o escopo deste trabalho busca analisar metadados de vídeos do Youtube a partir da perspectiva do padrão IEEE-LOM. 


\section{Método}

Inicialmente, esta pesquisa consistiu na verificação de quais metadados da categoria educacional do padrão IEEE-LOM seriam relevantes para o contexto abordado. Considerando as limitações da plataforma do Youtube e os objetivos desta pesquisa, foram selecionados os seguintes metadados para análise: a) tipo de interatividade; b) faixa-etária; c) tipo de recurso educacional; d) densidade semântica; e) dificuldade. $\mathrm{O}$ idioma dos vídeos selecionados foi o português do Brasil.

Com relação ao tipo de recurso educacional, foi utilizado também alguns vocabulários da extensão CLEO (Customized Learning Experience Online) [Taliesin 2003]. A extensão, para a categoria educacional, adiciona vocabulários alternativos para representar os recursos de aprendizagem e novos elementos.

A partir dos metadados selecionados do IEEE-LOM, o passo seguinte foi a composição da base de dados a ser avaliada. A fim de delimitar a investigação, foram utilizados os critérios de inclusão e exclusão dos vídeos, conforme a Tabela 1:

Tabela 1. Critérios de inclusão e exclusão dos vídeos.

\begin{tabular}{l|l}
\hline Critério & \multicolumn{1}{c}{ Descrição } \\
\hline \multirow{3}{*}{ Inclusão } & - Possuir temática na área de programação de computadores \\
& - Pertencer à categoria educacional \\
& - Ter tempo médio entre 4 e 20 minutos \\
\hline \multirow{3}{*}{ Exclusão } & - Estar em um idioma diferente do português (Brasil) \\
& - Ser duplicado \\
& - Não possuir legendas \\
\hline
\end{tabular}

A busca pelos vídeos que compuseram a base foi realizada utilizando a API (Application Programming Interface) do Youtube ${ }^{3}$. Os vídeos da plataforma Youtube possuem, dentre outras características, a respectiva categoria a qual são enquadrados. Esta categoria é selecionada pelo autor do vídeo durante o carregamento na plataforma. Destaca-se que na busca padrão pela página Web do Youtube não é possível selecionar a categoria do vídeo, mas com a API, este e outros critérios podem ser explorados, auxiliando no desenho da base a ser construída para análise. Ao todo foram utilizados 16 temas para compor essa base, conforme relacionados na Tabela 2.

Tabela 2. Temas usados na busca de vídeos para a base.

\begin{tabular}{l|l}
\hline & Temas \\
\hline - Classes em Java & - Funções em C \\
- Entrada e saída em C & - Herança em Java \\
- Algoritmo de ordenação Bubble Sort & - Matrizes em C \\
- Estrutura de dados Fila em C & - Métodos em Java \\
- Estrutura de dados Pilha & - Polimorfismo em Java \\
- Estrutura de dados árvores AVL & - Ponteiros em C \\
- Estrutura de repetição em Python & - Tabelas em HTML \\
- Estruturas condicionais em Python & - Vetores em Python \\
\hline
\end{tabular}

Além das temáticas acima, com a API do Youtube foi possível realizar outros filtros que se tornaram relevantes para a criação da base de dados investigada. Com relação ao atributo categoria (videoCategoryId), restringiu-se somente por vídeos educacionais

\footnotetext{
${ }^{3}$ https://developers.google.com/youtube/v3
} 
(categoria indicada pelo ID 27). O atributo duração (videoDuration) foi definido com valor medium, referindo-se aos vídeos que possuem duração acima de quatro minutos e abaixo de 20 minutos. Este valor foi definido considerando os parâmetros apontados em [Almeida and Carvalho 2018]. Para cada tema foram selecionados cinco vídeos, utilizando o atributo maxResults para limitação das consultas.

Dessa maneira, a base inicial contou com 80 vídeos da categoria educacional e com duração entre 4 e 20 minutos, retornados de forma padrão pelo algoritmo de busca da API do Youtube. Após aplicar os critérios de exclusão, restaram 69 vídeos para posterior avaliação. Os vídeos foram distribuídos para especialistas, em sua maioria docentes, e estudantes da área de Tecnologia da Informação. Para cada vídeo, selecionou-se um discente e um especialista para avaliação, criando assim duas bases distintas. A distribuição para as bases foi feita de forma com que houvesse uma harmonia entre a quantidade de vídeos avaliadas por ambas.

Não somente, mas também as referidas bases de dados foram particionadas entre voluntários, que realizaram um treinamento acerca dos metadados de OA avaliados, e os que não receberam esse treinamento, totalizando quatro bases distintas. Os voluntários que receberam o treinamento responderam, para cada vídeo assistido, a faixa-etária indicada, o tipo de interatividade, os tipos de recursos de aprendizagem, além da dificuldade e densidade semântica, tendo um domínio maior sobre os conceitos respondidos a partir das definições do IEEE-LOM.

Tabela 3. Questionário acerca dos vídeos avaliados.

\begin{tabular}{|c|c|c|}
\hline Pergunta & Opções & Tipo \\
\hline Você é? & $\begin{array}{l}\text { Docente ou especialista; } \\
\text { Discente }\end{array}$ & Botão de Seleção \\
\hline Tipo de recurso de aprendizagem & $\begin{array}{l}\text { Analogia; Apresentação; } \\
\text { Avaliação; Declaração; } \\
\text { Definição; Demonstração; } \\
\text { Diagrama; Exemplo; } \\
\text { Exercício; Experiência; } \\
\text { Introdução; Narrativa; } \\
\text { Palestra; Problema; } \\
\text { Prova; Questionário; } \\
\text { Resumo; Simulação; } \\
\text { Slide; Tabela; Texto; } \\
\text { Visão Geral }\end{array}$ & Caixa de Seleção \\
\hline Densidade Semântica & $\begin{array}{l}\text { Muito Baixa; Baixa; Média; } \\
\text { Alta; Muito Alta }\end{array}$ & Botão de Seleção \\
\hline Dificuldade & $\begin{array}{l}\text { Muito Fácil; Fácil; Média; } \\
\text { Difícil; Muito Difícil }\end{array}$ & Botão de Seleção \\
\hline
\end{tabular}

Os voluntários que receberam o treinamento foram os primeiros submetidos à pesquisa. A partir da análise dos resultados, notou-se que a faixa-etária e o tipo de interatividade não sofreram variação. O motivo provável foi o escopo da pesquisa e a plataforma utilizada. Deste modo, para o segundo grupo de investigados estas variáveis não foram utilizadas. A Tabela 3 demonstra a estrutura do questionário aplicado para o segundo grupo, ao qual envolveu discentes e especialistas sem um treinamento sobre os metadados de OA. Para cada uma das perguntas do questionário, havia a definição sucinta dos conceitos tratados. Dos 69 vídeos selecionados, 8 não obtiveram respostas de discentes 
IX Congresso Brasileiro de Informática na Educação (CBIE 2020)

Anais do XXXI Simpósio Brasileiro de Informática na Educação (SBIE 2020)

e/ou especialistas, restando para análise 61 respostas.

Ademais, buscando complementar as bases de dados de especialistas e discentes, fez-se uso de duas APIs voltadas para o Youtube. Sendo assim, foi possível extrair os seguintes metadados dos vídeos: data de publicação, número de visualizações, comentários, curtidas, descurtidas, duração em segundos e legenda (gerada automaticamente pelo Youtube). Após coletadas, as respostas do questionário e os dados extraídos da API foram tabulados e armazenados em formato .csv (comma-separated values). A seguir, têm-se os experimentos executados e os resultados obtidos.

\section{Experimentos e Resultados}

A fim de analisar as correlações entre os metadados dos vídeos e os metadados da categoria educacional do padrão IEEE-LOM, fez-se uso da linguagem de programação Python, na versão 3.6.9. Com relação à exploração e análise dos dados, foram utilizadas: a biblioteca de computação científica Numpy ${ }^{4}$; a ferramenta de análise de dados Pandas ${ }^{5}$ e a interface de recursos léxicos NLTK (Natural Language Toolkit) [Bird et al. 2009]. Assim, esta seção divide-se em: i) pré-processamento, fase na qual os dados foram tratados; e ii) análise, fase da visualização e discussão dos resultados.

\subsection{Pré-Processamento dos Dados}

Inicialmente, foi conduzida uma busca por registros inválidos ou faltantes na base de dados (NaN rows). Tal busca não demonstrou nenhuma anomalia, o que garantiu a consistência da base. Em seguida, realizou-se uma visualização dos tipos de dados contidos nas colunas (classificados em numéricos ou textuais) e foram encontrados os seguintes problemas: a densidade semântica, dificuldade e data estavam armazenadas como texto. Sendo assim, as duas primeiras foram mapeadas para números, enquanto que a coluna de data foi convertida para o número de dias decorridos desde a publicação do vídeo até a realização desta pesquisa. A conversão da coluna de data de publicação para um tipo numérico foi útil na normalização das colunas que dependem ativamente da mesma, a saber: número de visualizações, comentários, curtidas e descurtidas. O processo consistiu em dividir o valor destas colunas em cada registro, pelo valor da data, fazendo com que tais valores fiquem ponderados pela data em que o vídeo foi publicado.

Outra etapa do pré-processamento consistiu na extração de informações úteis a partir da legenda de cada vídeo. Com o auxílio da interface NLTK, foi possível fazer as etapas de tokenize, remoção de palavras vazias (stop words) e stemming e, após isso, extrair: (i) a quantidade de palavras sem repetição, (ii) quantidade total de palavras, (iii) quantidade de substantivos sem repetição e (iv) quantidade total de substantivos. A partir desses dados criou-se duas novas variáveis definidas como sendo a razão entre (i) e (ii) e a razão entre (iii) e (iv), denotadas como ratiol e ratio2, respectivamente. Tais valores de ratio se mostraram úteis na exploração da relação entre as respostas dos questionários e as características extraídas de forma automática dos vídeos.

O último passo do pré-processamento consistiu em lidar com os tipos de recursos de aprendizagem, conforme listados na Tabela 3. Inicialmente, os recursos para cada registro estavam em formato de texto, concatenados por vírgula (para caso houvesse mais

\footnotetext{
${ }^{4}$ https://numpy.org/

${ }^{5}$ https://pandas.pydata.org/
} 
de um recurso presente no vídeo). Objetivando uma melhor análise, foram criadas 22 colunas para representar cada um dos recursos. Em cada registro, o valor da respectiva coluna do recurso recebeu valor 1 , em relação à presença daquele recurso ou 0 , em sua ausência. As colunas presentes na base de dados bem como seus tipos após a fase de pré-processamento encontram-se na Tabela 4. Com todas as colunas devidamente normalizadas e adequadas, seguiu-se para a análise destas informações.

Tabela 4. Colunas e tipos após o pré-processamento.

\begin{tabular}{ll}
\hline \multicolumn{1}{c}{ Colunas Numéricas } & Colunas Textuais \\
\hline $\begin{array}{l}\text { Densidade; dificuldade; visualizações; tipo de recurso educacional; } \\
\text { curtidas; descurtidas; duração; data de publicação (dias decorridos); }\end{array}$ & Legenda \\
ratio1, ratio2 e comentários; & \\
\hline
\end{tabular}

\subsection{Análise dos Resultados}

A busca pelas correlações entre as variáveis foi realizada por meio da extração do coeficiente de correlação de Pearson [Morettin and Bussab 2017]. A correlação de Pearson é calculada com a seguinte fórmula:

$$
\operatorname{corr}(X, Y)=\frac{\sum x_{\mathrm{i}} y_{\mathrm{i}}-n \overline{x y}}{\sqrt{\left(\sum x_{\mathrm{i}}^{2}-n \bar{x}^{2}\right)\left(\sum y_{\mathrm{i}}^{2}-n \bar{y}^{2}\right)}}
$$

Onde $\mathrm{X}$ e Y são os vetores dos dados a serem avaliados, sendo $\bar{x}$ e $\bar{y}$ suas respectivas médias aritméticas e $n$ o número de elementos em cada vetor. A correlação obedece a seguinte desigualdade: $-1 \leq \operatorname{corr}(X, Y) \leq 1$. Um resultado próximo de 0 indica ausência de correlação linear entre os vetores, enquanto um resultado próximo de 1 indica dependência linear positiva e por último, próximo a -1 indica dependência linear negativa. Para determinar se uma correlação é significativa, também é feita a comparação do indicador $p$-value com o nível de significância estabelecido. Considerando o valor de $p$, é possível aferir se o coeficiente de correlação é significativamente diferente de 0 .

Feitas estas considerações, foram gerados mapas de calor das correlações, que representam a magnitude distribuída entre os coeficientes de correlação presentes nas variáveis analisadas nos questionários dos especialistas e dos discentes sem treinamento prévio, conforme apresentado nas Figuras 1 e 2.

\begin{tabular}{|c|c|c|c|c|c|c|c|c|c|c|}
\hline & densidade & dificuldade & visualizacoes & curtidas & descurtidas & comentarios & duracao & data & ratio1 & ratio2 \\
\hline densidade & 1.00 & 0.46 & -0.23 & -0.15 & -0.21 & -0.07 & 0.27 & -0.10 & -0.21 & -0.25 \\
\hline dificuldade & 0.46 & 1.00 & -0.19 & -0.21 & -0.23 & -0.20 & -0.06 & 0.03 & 0.17 & 0.12 \\
\hline visualizacoes & -0.23 & -0.19 & 1.00 & 0.93 & 0.80 & 0.63 & 0.19 & 0.37 & -0.22 & -0.10 \\
\hline curtidas & -0.15 & -0.21 & 0.93 & 1.00 & 0.73 & 0.74 & 0.33 & 0.18 & -0.28 & -0.11 \\
\hline descurtidas & -0.21 & -0.23 & 0.80 & 0.73 & 1.00 & 0.67 & 0.17 & 0.21 & -0.23 & -0.02 \\
\hline comentarios & -0.07 & -0.20 & 0.63 & 0.74 & 0.67 & 1.00 & 0.18 & -0.07 & -0.14 & 0.04 \\
\hline duracao & 0.27 & -0.06 & 0.19 & 0.33 & 0.17 & 0.18 & 1.00 & -0.21 & -0.84 & -0.51 \\
\hline data & -0.10 & 0.03 & 0.37 & 0.18 & 0.21 & -0.07 & -0.21 & 1.00 & 0.12 & -0.01 \\
\hline ratio1 & -0.21 & 0.17 & -0.22 & -0.28 & -0.23 & -0.14 & -0.84 & 0.12 & 1.00 & 0.72 \\
\hline ratio2 & -0.25 & 0.12 & -0.10 & -0.11 & -0.02 & 0.04 & -0.51 & -0.01 & 0.72 & 1.00 \\
\hline
\end{tabular}

Figura 1. Mapa de calor das correlações da base dos especialistas. 


\begin{tabular}{|c|c|c|c|c|c|c|c|c|c|c|}
\hline & densidade & dificuldade & visualizacoes & curtidas & descurtidas & comentarios & duracao & data & ratio1 & ratio2 \\
\hline densidade & 1.00 & 0.45 & -0.13 & -0.10 & -0.10 & -0.09 & 0.27 & -0.14 & -0.30 & -0.43 \\
\hline dificuldade & 0.45 & 1.00 & -0.16 & -0.14 & -0.13 & -0.12 & 0.32 & -0.07 & -0.32 & -0.34 \\
\hline visualizacoes & -0.13 & -0.16 & 1.00 & 0.93 & 0.80 & 0.63 & 0.19 & 0.37 & -0.22 & -0.10 \\
\hline curtidas & -0.10 & -0.14 & 0.93 & 1.00 & 0.73 & 0.74 & 0.33 & 0.18 & -0.28 & -0.11 \\
\hline descurtidas & -0.10 & -0.13 & 0.80 & 0.73 & 1.00 & 0.67 & 0.17 & 0.21 & -0.23 & -0.02 \\
\hline comentarios & -0.09 & -0.12 & 0.63 & 0.74 & 0.67 & 1.00 & 0.18 & -0.07 & -0.14 & 0.04 \\
\hline duracao & 0.27 & 0.32 & 0.19 & 0.33 & 0.17 & 0.18 & 1.00 & -0.21 & -0.84 & -0.51 \\
\hline data & -0.14 & -0.07 & 0.37 & 0.18 & 0.21 & -0.07 & -0.21 & 1.00 & 0.12 & -0.01 \\
\hline ratio1 & -0.30 & -0.32 & -0.22 & -0.28 & -0.23 & -0.14 & -0.84 & 0.12 & 1.00 & 0.72 \\
\hline ratio2 & -0.43 & -0.34 & -0.10 & -0.11 & -0.02 & 0.04 & -0.51 & -0.01 & 0.72 & 1.00 \\
\hline
\end{tabular}

Figura 2. Mapa de calor das correlações da base dos discentes.

É possível perceber que somente as correlações das primeiras duas colunas (densidade e dificuldade) diferem entre si nas figuras, isso ocorre pois as outras colunas são informações dos vídeos e não das respostas dos questionários. Também constata-se que as principais discrepâncias entre as respostas de especialistas e estudantes, em termos de correlação, se deu entre os ratios, dificuldade e densidade, visto que em outras colunas as diferenças foram menos bruscas. Nota-se uma correlação positiva moderada entre dificuldade e densidade, a qual se mantém praticamente estável entre os dois questionários (respectivamente, 0,46 e 0,45), com p-value menor que 0,05.

Outras considerações pertinentes referem-se à duração e densidade. Analisando os resultados obtidos, é encontrado um coeficiente de correlação $=0,27$ e $p$-value $=0,01$. Este índice foi a segunda correlação positiva mais alta, demonstrando a existência da mesma visão para os dois públicos quanto ao entendimento do tempo de duração e sua densidade semântica. Apesar disso, a mesma é tida como correlação fraca. Considerando somente os estudantes, duração e dificuldade também possuiu correlação fraca e significativa de 0,31 .

As correlações entre as características dos vídeos, tais como visualizações, curtidas, descurtidas e comentários em relação aos indicadores densidade e dificuldade semântica são consideradas, em maioria, como bem fracas e negativas. Isso indica associação desprezível entre as variáveis, contudo, os resultados não são considerados como significativos, haja vista $p$-value maior que 0,05 para todos os casos.

Todavia, ao analisar somente os dados oriundos da bases dos voluntários que receberam treinamento sobre os metadados de IEEE-LOM, os resultados apresentam uma diferença. A exemplo disso, para os especialistas, duração e densidade possuíram correlação de 0,53 e $p$-value $=0,001$, considerado como muito significativa. Para dificuldade e densidade, obteve-se coeficiente de correlação $=0,39$ e $p$-value $=0,01$. Quanto aos estudantes, o coeficiente atingiu correlação forte positiva $(0,74)$ e significativa para dificuldade e densidade, com valor de $p$ menor que 0,01 . Até mesmo para duração e densidade o índice foi elevado (0,57 significativo). Ressalta-se que para as características dos vídeos, as correlações permaneceram com níveis de significância maiores que 0,05 em ambos os perfis.

Em relação à classificação geral, além das correlações, também foi analisada a 
distribuição dos tipos de recursos de aprendizagem no questionário do especialista (Figura 3(a)) e do aluno (Figura 3(b)). A visualização dos tipos de recursos de aprendizagem permite identificar que os recursos avaliação, prova e questionário não foram marcados em nenhum dos questionários. Ademais, verifica-se uma forte prevalência dos recursos apresentação, demonstração e exemplo em ambos os questionários.

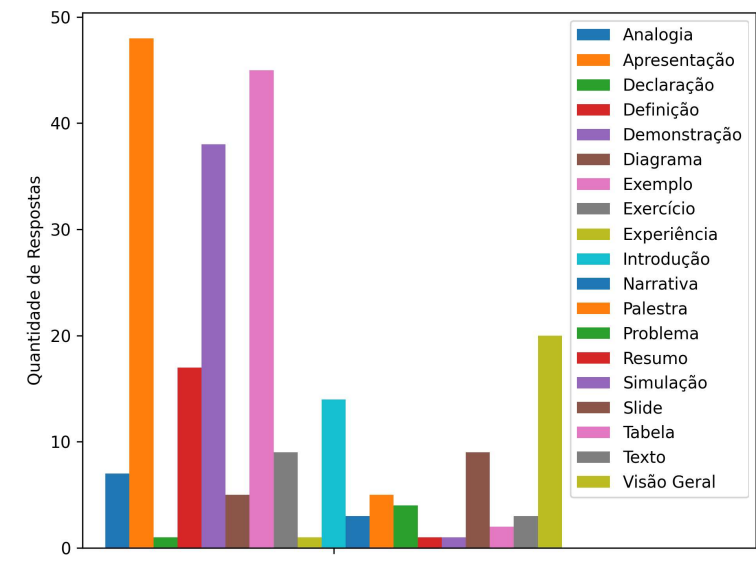

(a) Questionário do especialista.

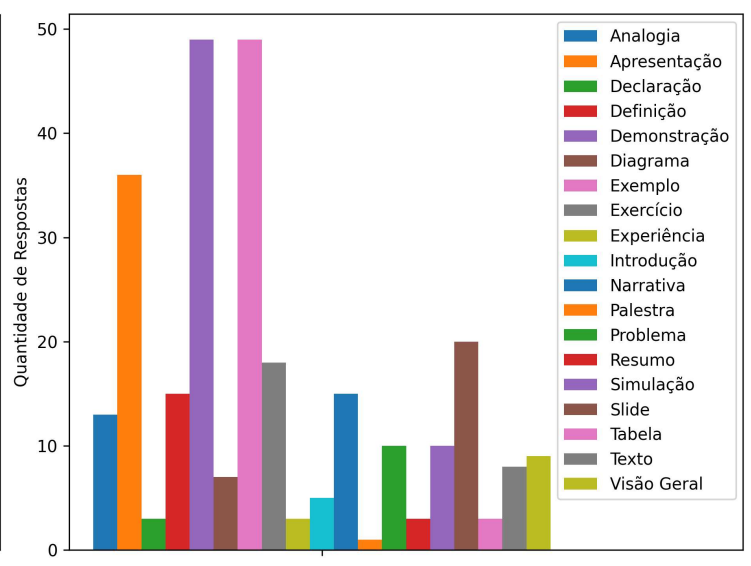

(b) Questionário do aluno.

Figura 3. Distribuições dos recursos de aprendizagem no questionário do especialista e do aluno, respectivamente.

\section{Considerações Finais}

O presente estudo objetivou evidenciar correlações entre os metadados de 61 vídeos da plataforma Youtube e os metadados de OA do IEEE-LOM (com apoio da extensão CLEO). Os resultados apontaram correlações moderadas entre dificuldade e densidade para a base geral de especialistas e estudantes. Quando comparados com os metadados fornecidos pelas APIS do Youtube, foi possível encontrar uma correlação fraca da frequência de substantivos da narração de vídeos com dificuldade e densidade semântica. Notou-se também que a frequência de substantivos em vídeos possui correlação com os metadados de densidade semântica e dificuldade. Também foram evidenciados os recursos de aprendizagem mais identificados em vídeos da plataforma.

Os resultados apresentados neste trabalho limitam-se à análise de vídeos no âmbito de programação de computadores. A base analisada apresentou uma tendência à consideração de que quanto mais denso o vídeo, mais difícil será a aprendizagem por esse material, indicando que pode haver uma relação de causa-efeito entre as duas variáveis, justificado com maior ênfase pelo coeficiente de correlação do ponto de vista dos discentes voluntários com conhecimento prévio acerca do IEEE-LOM. Também observou a correlação negativa entre ratio2 e densidade, o que pode indicar que vídeos com maior repetição de substantivos (e, portanto, maior ratio2) tenderão a ser classificados como menos densos. Tais resultados são alguns exemplos que encorajam a novas investigações buscando causalidade entre metadados do IEEE-LOM e os fornecidos pelas APIs para auxiliar no processo de recomendação de tais recursos como materiais educacionais. 
IX Congresso Brasileiro de Informática na Educação (CBIE 2020)

Anais do XXXI Simpósio Brasileiro de Informática na Educação (SBIE 2020)

\section{Referências}

Almeida, C. d. M. e. and Carvalho, N. A. d. (2018). Avaliação da duração das videoaulas na perspectiva dos alunos do consórcio cederj. In Anais do vigésimo quarto CIAED Congresso Internacional ABED de Educação a Distância.

Andriani, A., Akib, E., and Ma'ruf, A. (2018). The use of spotify application to improve students ability in listening through english song. Journal of Computer Interaction in Education (JCIE), 1(2):120-131.

Barrére, E., Souza, J., Vitor, M., and Almeida, M. (2018). Recomendação automática de videoaulas no moodle. Brazilian Symposium on Computers in Education (Simpósio Brasileiro de Informática na Educação - SBIE), 29(1):1613.

Bhabad, D. T., Therese, S., and Gedam, M. (2017). Multimedia based information retrieval approach based on asr and ocr and video recommendation system. In 2017 International Conference on Current Trends in Computer, Electrical, Electronics and Communication (CTCEEC), pages 1168-1172.

Bird, S., Klein, E., and Loper, E. (2009). Natural language processing with Python: analyzing text with the natural language toolkit. "O'Reilly Media, Inc.".

Cedefop (2014). Terminology of European education and training policy: A selection of 130 terms (2nd ed.). Luxembourg: Publications Office.

De Lima Medeiros, S. F. and Pansanato, L. (2015). Estudo das preferências de alunos e professores sobre videoaula para identificar requisitos de software para ferramentas de produçao. In Brazilian Symposium on Computers in Education (Simpósio Brasileiro de Informática na Educação-SBIE), volume 26, page 219.

De Medio, C., Limongelli, C., Marani, A., and Taibi, D. (2019). Retrieval of educational resources from the web: A comparison between google and online educational repositories. In International Conference on Web-Based Learning, pages 28-38. Springer.

García-Floriano, A., Ferreira-Santiago, A., Yáñez-Márquez, C., Camacho-Nieto, O., Aldape-Pérez, M., and Villuendas-Rey, Y. (2017). Social web content enhancement in a distance learning environment: intelligent metadata generation for resources. International Review of Research in Open and Distributed Learning, 18(1):161-176.

McClelland, M. (2003). Metadata standards for educational resources. Computer, 36(11):107-109.

Morettin, P. A. and Bussab, W. O. (2017). Estatística básica. Saraiva Educação SA.

Pal, S., Pramanik, P. K. D., Majumdar, T., and Choudhury, P. (2019). A semi-automatic metadata extraction model and method for video-based e-learning contents. Education and Information Technologies, 24(6):3243-3268.

Taliesin, B. (2003). Cleo extensions to the ieee learning object metadata.

Westerhout, E., Monachesi, P., Markus, T., and Posea, V. (2010). Enhancing the learning process: Qualitative validation of an informal learning support system consisting of a knowledge discovery and a social learning component. In Wolpers, M., Kirschner, P. A., Scheffel, M., Lindstaedt, S., and Dimitrova, V., editors, Sustaining TEL: From Innovation to Learning and Practice, pages 374-389, Berlin, Heidelberg. Springer Berlin Heidelberg. 